\title{
Towards an intelligent tool based on Concept Maps for an Automated Meaningful Learning
}

\author{
Lorenzo Moreno $^{1}$, Evelio J. González ${ }^{1}$, Beatrice Popescu ${ }^{1}$, José D. Piñeiro ${ }^{1}$ \\ and Claudia L. Oliveira Groenwald ${ }^{2}$ \\ 1 Dpto. Ingeniería de Sistemas y Automática y Arquitectura y Tecnología \\ de Computadores. Universidad de La Laguna, CP 38207. \\ Tenerife, Spain, lmoreno@ull.es \\ 2 Faculdade de Matemática, Universidade Luterana do Brasil
}

\section{Summary}

The goal of this work is the development of an intelligent tool that would help teachers and students in order to improve the learning process, making use of a variation of the concept map paradigm. The authors propose a novelty in their use for determining the actual student status, that is, what a student currently knows, which concepts a student needs to revise, etc.

The proposed tool describes the subject to learn from an evolution of conceptual maps, called Pedagogical Concept Instructional Graph (PCIG), generated by the teacher. A PCIG does not order the concepts following arbitrary relationships among them as in the case of a general concept map, but the concepts are placed depending on the logical order in which they are presented to the student. After that, the expert system makes use of an adaptive test, generating several questions that deal with those concepts included in the PCIG.

From each student's answers, it can be obtained a personalized PCIG describing what the student knows a priori about the subject to learn. Comparing both models the expert system will determine a learning path, based in cases and personalized for groups of students presenting similar characteristics.

There are two points of view in order to implement the proposed tool: the learner that must pass the tests and view the contents related to some concepts and the teacher that creates the concepts, the test questions, the contents, defines works and assigns them to the learners.

- The entry point is a PCIG describing the relations between the concepts. An edge [concept1, concept2] denotes that concept1 
depends on concept2, thus a learner couldn't know concept1 without knowing concept 2 .

- $\quad$ The teacher should create the questions specifying those parameters found in each Bayesian-type networks, since the adaptive tests have been implemented following that approach.

- $\quad$ Definition of the initial concepts that the learner should already know from previous completed works and the objective concepts (those that the learner should take and pass a test in order to complete the task).

- $\quad$ Tracking the state of each learner by the teacher.

- Create contents related to each concept.

- A student can only choose those concepts that he has not already passed, and do not depend on not-passed concepts.

The proposed tool has been implemented as a web-based application written in Java tested for Tomcat application server and using a MySQL database. A test algorithm is defined and implementations for the following actions are provided. First, the way the next question is chosen from the list of available questions. The available questions are questions related to the currently testing concept and that is not related to concepts that the learner hasn't passed yet. The way the points obtained modify if he responded correctly or not at the previous question when the test stops. Currently this is implemented using an ANTLR parser and in code using the Bayesian algorithm and it is being validated with real users, in particular, in the field of Maths in secondary education. This validation will allow the teachers to carry out a realistic and personalized teaching planning, based on meaningful learning. For the inclusion of mathematical formulae, the tool includes DragMath Editor for MathML.

\section{References}

1. Novak, J.D. Clarify with concept maps: A tool for students and teachers alike. The Science Teacher. 1991, 58(7), 45-49.

2. Michael J. In pursuit of meaningful learning. Adv. Physiol. Educ. 2001, 25: 145-158. 